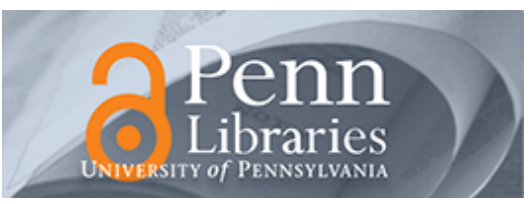

University of Pennsylvania

ScholarlyCommons

September 2000

\title{
Fractionalization Methods and their Applications to Radiation and Scattering Problems
}

\author{
Nader Engheta \\ University of Pennsylvania, engheta@seas.upenn.edu
}

Follow this and additional works at: https://repository.upenn.edu/ese_papers

\section{Recommended Citation}

Nader Engheta, "Fractionalization Methods and their Applications to Radiation and Scattering Problems ", . September 2000.

Copyright 2000 IEEE. Reprinted from International Conference on Mathematical Methods in Electromagnetic Theory 2000 (MMET 2000) Volume 1, pages 34-40.

Publisher URL: http://ieeexplore.ieee.org/xpl/tocresult.jsp?isNumber=19211

This material is posted here with permission of the IEEE. Such permission of the IEEE does not in any way imply IEEE endorsement of any of the University of Pennsylvania's products or services. Internal or personal use of this material is permitted. However, permission to reprint/republish this material for advertising or promotional purposes or for creating new collective works for resale or redistribution must be obtained from the IEEE by writing to pubs-permissions@ieee.org. By choosing to view this document, you agree to all provisions of the copyright laws protecting it.

This paper is posted at ScholarlyCommons. https://repository.upenn.edu/ese_papers/15

For more information, please contact repository@pobox.upenn.edu. 


\title{
Fractionalization Methods and their Applications to Radiation and Scattering Problems
}

\author{
Abstract \\ Exploring the possible links between the mathematical field of fractional calculus and the \\ electromagnetic theory has been one of the topics of our research interests in recent years. We have \\ studied the possibility of bringing the tools of fractional calculus and electromagnetic theory together, \\ and have explored and developed the topic of fractional paradigm in electromagnetic theory (see e.g., \\ [1-10]). Fractional calculus is a branch of mathematics that addresses the mathematical properties of \\ operation of fractional differentiation and fractional integration - operators involving derivatives and \\ integrals to arbitrary non-integer orders (see e.g., [11-13]). In our study in recent years, we have applied \\ the tools of fractional calculus in various problems in electromagnetic fields and waves, and have \\ obtained interesting results that highlight certain notable features and promising potential applications of \\ these operators in electromagnetic theory [1-10]. Moreover, since fractional derivatives/integrals are \\ effectively the result of fractionalization of differentiation and integration operators, we have investigated \\ the notion of fractionalization of some other linear operators in electromagnetic theory. Searching for \\ such operator fractionalization has led us to interesting solutions in radiation and scattering problems.

\section{Comments} \\ Copyright 2000 IEEE. Reprinted from International Conference on Mathematical Methods in \\ Electromagnetic Theory 2000 (MMET 2000) Volume 1, pages 34-40. \\ Publisher URL: http://ieeexplore.iee. $0 \mathrm{rg} / \mathrm{xpl} /$ tocresult.jsp?isNumber=19211 \\ This material is posted here with permission of the IEEE. Such permission of the IEEE does not in any way \\ imply IEEE endorsement of any of the University of Pennsylvania's products or services. Internal or \\ personal use of this material is permitted. However, permission to reprint/republish this material for \\ advertising or promotional purposes or for creating new collective works for resale or redistribution must \\ be obtained from the IEEE by writing to pubs-permissions@ieee.org. By choosing to view this document, \\ you agree to all provisions of the copyright laws protecting it.
}




\title{
FRACTIONALIZATION METHODS AND THEIR APPLICATIONS TO RADIATION AND SCATTERING PROBLEMS
}

\author{
Nader Engheta \\ University of Pennsylvania \\ Moore School of Electrical Engineering \\ Philadelphia, Pennsylvania 19104, U.S.A. \\ Tel: +1 (215) 898-9777, Fax: +1 (215) 573-2068 \\ E-mail: engheta@ee.upenn.edu \\ Web: http://www.ee.upenn.edu/ engheta/
}

\section{INTRODUCTION:}

Exploring the possible links between the mathematical field of fractional calculus and the electromagnetic theory has been one of the topics of our research interests in recent years. We have studied the possibility of bringing the tools of fractional calculus and electromagnetic theory together, and have explored and developed the topic of fractional paradigm in electromagnetic theory (see e.g., [1-10]). Fractional calculus is a branch of mathematics that addresses the mathematical properties of operation of fractional differentiation and fractional integration operators involving derivatives and integrals to arbitrary non-integer orders (see e.g., [11-13]). In our study in recent years, we have applied the tools of fractional calculus in various problems in electromagnetic fields and waves, and have obtained interesting results that highlight certain notable features and promising potential applications of these operators in electromagnetic theory [1-10]. Moreover, since fractional derivatives/integrals are effectively the result of fractionalization of differentiation and integration operators, we have investigated the notion of fractionalization of some other linear operators in electromagnetic theory. Searching for such operator fractionalization has led us to interesting solutions in radiation and scattering problems.

\section{"FRACTIONAL SOLUTIONS" IN RADIATION AND SCATTERING PROBLEMS}

Let us consider the time-harmonic Maxwell equations $\nabla \times \boldsymbol{E}=i \omega \boldsymbol{B}, \nabla \times \boldsymbol{H}=\boldsymbol{J}-i \omega \boldsymbol{D}$, $\nabla \cdot \boldsymbol{D}=\rho$, and $\nabla \cdot \boldsymbol{B}=0$ where the time dependence $e^{-i \omega t}$ is assumed, and the electric current density $\boldsymbol{J}$ may be considered either as a primary current source (in radiation problems) or as a secondary, induced current (in scattering problems). The continuity equation is of course satisfied as $\nabla \cdot \boldsymbol{J}=-i \omega \rho$. Now let us choose a linear operator $\underline{\underline{\boldsymbol{L}}}$ from a class of linear operators (or mappings) where the domain and range of any linear operator of this class are similar to each other and have the same dimensions. In other words, the linear operator $\underline{\underline{L}}$ should map an element from the space $C^{n}$ into generally another element in the space $C^{n}$. That is

$$
\underline{\underline{L}}: C^{n} \rightarrow C^{n}
$$


where $C^{n}$ is an n-dimensional vector space over the field of complex numbers. One can then suggest a "fractional" operator $\underline{\underline{\boldsymbol{L}}}^{\nu}$, where the fractionalization parameter $v$ can be a noninteger real number (or in some circumstances even complex number), using some techniques one of which is reviewed in [5]. Such a fractional operator $\underline{\underline{L}}^{v}$, which also maps the n-dimensional space into the $n$-dimensional space

$$
\underline{\underline{L^{\nu}}}: C^{n} \rightarrow C^{n}
$$

has the semigroup properties, i.e., (1) for $v \rightarrow 0$, the fractional operator $\underline{\underline{L}}^{v}$ approaches the unit operator, $\underline{\underline{L}}^{\nu} \rightarrow \underline{\underline{L}}^{o}=\underline{\underline{I}}$; (2) for $v \rightarrow 1$, the operator $\underline{\underline{L}}^{\nu}$ approaches the original operator $\underline{\underline{\boldsymbol{L}}}$, $\underline{\underline{L}}^{\nu} \rightarrow \underline{\underline{\boldsymbol{L}}}=\underline{\underline{\boldsymbol{L}}}$; and (3) for two parameters $v_{1}$ and $v_{2}$ we will have $\underline{\underline{\boldsymbol{L}}}^{\nu_{1}} \circ \underline{\underline{L}}^{\nu_{2}}=\underline{\underline{\boldsymbol{L}}}^{\nu_{1}+\nu_{2}}$. If the vector fields $\boldsymbol{E}_{o}, \boldsymbol{H}_{o}, \boldsymbol{B}_{o}$, and $\boldsymbol{D}_{o}$ are the solutions to the time-harmonic Maxwell equations with the current density $J_{o}$, one can pose the following question: If we apply the fractional operator $\underline{\underline{L}}^{\nu}$ on these vector solutions, under which condition(s) will the resulting vector functions, $\underline{\underline{L}}^{v} \boldsymbol{E}_{o}$, $\underline{\underline{L}}^{\nu} \boldsymbol{H}_{o}, \underline{\underline{L}}^{\nu} \boldsymbol{B}_{o}$, and $\underline{\underline{L}}^{\nu} \boldsymbol{D}_{o}$ satisfy the Maxwell equations with the new current density $\underline{\underline{L}}^{\nu} \boldsymbol{J}_{o}$ ? One can observe that if the fractional operator $\underline{\underline{L}}^{v}$ and the curl operator $\nabla \times$ commute, i.e., if

$$
\underline{\underline{L}}^{\nu} \nabla \times=\nabla \times \underline{\underline{L}}^{\nu}
$$

one will then have

$$
\begin{aligned}
& \nabla \times\left(\underline{\underline{L}}^{\nu} \boldsymbol{E}_{o}\right)=i \omega\left(\underline{\underline{\boldsymbol{L}}}^{\nu} \boldsymbol{B}_{o}\right) \\
& \nabla \times\left(\underline{\underline{\boldsymbol{L}}}^{\nu} \boldsymbol{H}_{o}\right)=\underline{\underline{L}}^{\nu} \boldsymbol{J}_{o}-i \omega\left(\underline{\underline{L}}^{\nu} \boldsymbol{D}_{o}\right)
\end{aligned}
$$

which indicates that the new set of vector functions $\underline{\underline{L}}^{v} E_{o}, \underline{\underline{L}}^{v} \boldsymbol{H}_{o}, \underline{\underline{L}}^{v} B_{o}$, and $\underline{\underline{L}}^{v} D_{o}$ does satisfy the Maxwell equations with the current density $\underline{\underline{L}}^{\nu} \boldsymbol{J}_{\boldsymbol{o}}$. What this implies is that if we know a set of solutions for an electromagnetic problem with a given current source $\boldsymbol{J}_{\boldsymbol{o}}$, we will be able to find the set of solutions to the related problem in which the current source is expressed as $\underline{\underline{L}}^{v} \boldsymbol{J}_{o}$ by just applying the fractional operator $\underline{\underline{L}}^{\nu}$ on the solutions of the first problem. This can offer interesting mathematical applications in the treatment of certain electromagnetic problem. As some specific cases, we have considered the operators of fractional integration, fractional integration, and fractional curl to show some of the interesting features of this treatment of certain radiation and scattering problems. $[1,4,5,6,7,9]$ In the following section, we present an example of application of the fractional operator in a scattering problem.

\section{FRACTIONAL OPERATION AND PHYSICAL OPTICS APPROXIMATION}

Let us consider a flat rectangular patch with certain surface impedance boundary conditions (e.g., a perfectly electric conducting (PEC) rectangular patch). This obstacle is being illuminated by a monochromatic electromagnetic wave. The incident and the scattered fields are denoted by 
$\boldsymbol{E}^{i n c}(x, y, z)$ and $\boldsymbol{E}^{\text {scar. }}(x, y, z)$, respectively. Let us now apply the fractional differintegration operator (with respect to a (dimensionless) Cartesian coordinate, say $k_{o} z$, and with a general fractional order $v$ on the incident and the scattered fields. That is

$$
\begin{aligned}
& \boldsymbol{E}^{i n c . v}(x, y, z)={ }_{-\infty} D_{k_{o} z}^{v} E^{i n c}(x, y, z) \\
& \boldsymbol{E}^{\text {scat.,v}}(x, y, z)={ }_{-\infty} D_{k_{o} z}^{v} E^{s c a t .}(x, y, z)
\end{aligned}
$$

where ${ }_{-\infty} D_{k_{0} z}^{v}$ indicates the operation of fractional differintegration. For the definition of fractional integration, we use the Riemann-Liouville fractional integration [11] which is written as

$$
{ }_{a} D_{z}^{v} f(z) \equiv \frac{1}{\Gamma(-v)} \int_{a}^{z}(z-u)^{-v-1} f(u) d u \quad \text { for } \operatorname{Re}(v)<0
$$

If $\operatorname{Re}(v)>0$, one will need to choose an integer $m$ in the expression ${ }_{a} D_{z}^{v} f(z) \equiv \frac{d^{m}}{d z^{m}}{ }_{a} D_{z}^{v-m} f(z)$ such that $\operatorname{Re}(v-m)<0$. Then the above definition can be used to evaluate ${ }_{a} D_{z}^{v-n} f(z)[11]$.

We ask the following question: Can the resulting field, $\boldsymbol{E}^{\text {scat., }}(x, y, z)$, be shown to be approximately the field that would be scattered from another rectangular patch with the same size and shape but with a different surface impedance boundary condition that would be determined from the knowledge of surface impedance of the original scatterer and the fractional parameter $v$ ? The results of our preliminary theoretical study have shown that, under certain circumstances and approximations (such as physical optics approximation), the answer to the above question is positive for the case of finite-size flat obstacles. In order to express the relationship for the impedance transformation, we first describe below the case of an infinitely extent flat plate.

Let us assume an infinitely extent flat plate with a specified isotropic uniform surface impedance boundary condition, $\underline{\underline{Z}}_{s o}$. This requires that the tangential components of the total electric and magnetic fields on the surface of this infinite plate be related as $\boldsymbol{E}_{t}^{\text {iout }}=\underline{Z}_{\underline{\underline{Z}}} \boldsymbol{H}_{t}^{\text {total }}$. (See Fig. 1). A Cartesian coordinate system $(x, y, z)$ is used here. For the sake of brevity of discussion here, we consider the transverse electric (TE) case for the incident electromagnetic wave illuminating this plate (i.e. the vector $\boldsymbol{E}^{i n c}$ is polarized along the y axis). As shown in Fig. 1, the angle of incidence is chosen to be $\theta_{i}$ (with respect to the unit vector normal to the surface) and the angle of tilt of the plate (with respect to the $x$-axis) is taken to be $\theta_{o}$. Obviously the reflected plane wave can be easily written in terms of the reflection coefficient, $R_{o}$, which can be explicitly given as a function of the surface impedance $\underline{\underline{Z}}_{s o}$. Since we have assumed that the surface impedance is isotropic and uniform, one can write $E_{y}^{\text {total }}=Z_{s o} H_{t}^{\text {total }}$, where $Z_{s o}$ denotes the surface impedance on this flat boundary, and $\boldsymbol{H}_{t}^{\text {total }}$ indicates the total tangential magnetic field on the surface. Now 
we take the fractional differintegral of the total electric field $\boldsymbol{E}^{\text {total }}=\boldsymbol{E}^{\text {inc }}+\boldsymbol{E}^{\text {ref }}$ with respect to variable $k_{o} z$ and with fractional order $v$. We have

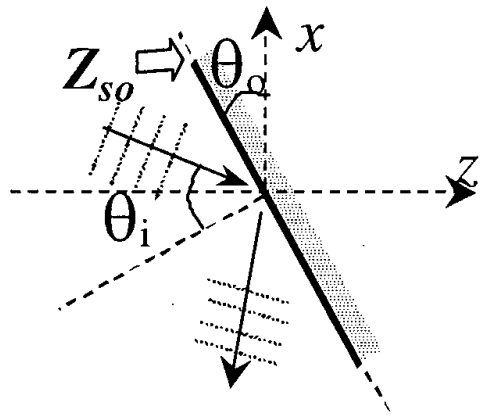

A

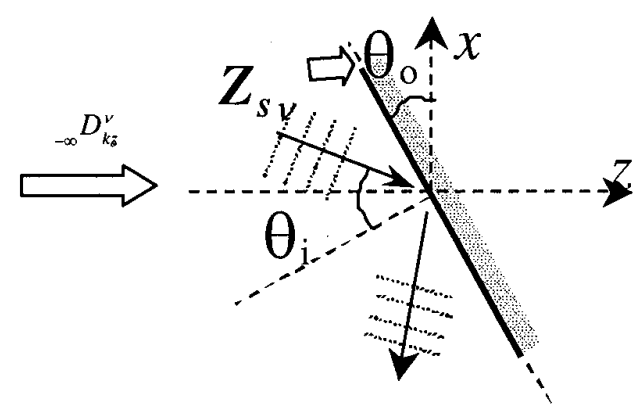

B

Fig. 1. Panel A depicts the problem of reflection of a TE incident plane wave from an infinitely extent flat boundary with the isotropic uniform surface impedance $Z_{s o}$. (The electric vector is parallel with the $y$ axis.) When the incident and reflected plane waves in such a problem are fractionally differintegrated with order $v$ with respect to the $z$ variable, the resulting functions can be interpreted as the new incident and new reflected waves in another problem (Panel B) with infinite flat boundary but with a differing surface impedance $Z_{s v}$. The relationship between $Z_{s o}, Z_{s v}$ and other relevant parameters is given in text.

$$
\begin{aligned}
E^{\text {total }, \nu} & ={ }_{-\infty} D_{k_{o} z}^{v} E^{\text {total }}==_{-\infty} D_{k_{o} z}^{v} E^{i n c}+_{-\infty} D_{k_{o} z}^{v} E^{r e f}= \\
& =E_{o} \hat{y}\left[\cos ^{\nu}\left(\theta_{i}-\theta_{i}\right) e^{i k_{o} z \cos \left(\theta_{i}-\theta_{o}\right)-i k_{o} x \sin \left(\theta_{i}-\theta_{o}\right)+i v \frac{\pi}{2}}+R_{o} \cos ^{\nu}\left(\theta_{i}+\theta_{i}\right) e^{-i k_{\theta} z \cos \left(\theta_{i}+\theta_{o}\right)-i k_{o} x \sin \left(\theta_{i}+\theta_{o}\right)-i v \frac{\pi}{2}}\right]
\end{aligned}
$$

The resulting total field, which can be shown to indeed satisfy the Maxwell equations and thus represent electromagnetic fields, consists of a new incident field, ${ }_{-\infty} D_{k_{o} z}^{v} E^{\text {inc }}$, that may be regarded as an incident plane wave with the incidence angle $\theta_{i}$ illuminating the plate with the tilt angle $\theta_{o}$, and the new reflected wave, ${ }_{-\infty} D_{k_{0} z}^{\nu} E^{r e f}$, that can be interpreted as the corresponding reflected plane wave. The tilt angle of the plate and the incidence angle remain as $\theta_{o}$ and $\theta_{i}$, respectively. However, the new reflection coefficient, which relates the new incident plane wave with the new reflected plane wave is different from $R_{o}$, and is given as

$$
R_{v}=R_{o} e^{-i v \pi} \frac{\cos ^{v}\left(\theta_{i}+\theta_{i}\right)}{\cos ^{v}\left(\theta_{i}-\theta_{i}\right)}
$$


Following a series of mathematical steps, for the TE case we can show that the new plate, which would provide such a reflection coefficient, should have a new surface impedance, denoted as $Z_{s v}$, that can be explicitly written as

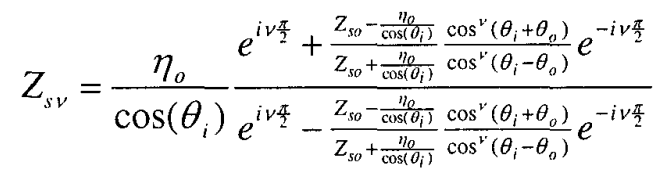

A similar approach can be used to obtain the corresponding expression for the TM case. The above results demonstrate that when the plane wave reflected from an infinite flat plate with the surface impedance $Z_{s o}$ is fractionally differintegrated (with the order $v$ ) the result can be interpreted as a plane wave that would have been reflected if the infinite plate (with the same tilt angle $\theta_{o}$ and the same angle of incidence $\theta_{i}$ for the incoming plane wave) had had a differing surface impedance $Z_{s v}$ that can be explicitly given in terms of all the quantities involved.

Although the example of reflection of plane wave from an infinite flat boundary with uniform surface impedance is an idealized example and the mathematical connections via fractional calculus for the infinite flat plates is rather formal, our preliminary analysis has shown that for the finite-size flat plates the operation of fractional differintegration may be quite useful to link and relate approximately the scattered fields for two separate problems with finite-size flat plates of differing surface impedances. To demonstrate this point, let us now return to our example of flat rectangular patch, and consider the problem of electromagnetic wave scattering from a rectangular, flat, thin, perfectly electric conducting (PEC) plate when the incoming plane wave is normally incident on this patch. Using the physical optics (PO) approximation, the scattered field can be easily obtained. Then we apply the fractional differintegration operator (with order $v$ ) on the far-zone scattered electric field in the region in front of the illuminated side of the rectangular plate. We then use the impedance transformation given in Eq. (9) to find $Z_{s v}$, and afterwards using the PO approximation we find the scattered fields from the rectangular plate with the same size and shape but with this new surface impedance $Z_{s v}$. Our analysis has shown that the result of fractional differintegration of the scattered field from the PEC rectangular plate is approximately similar to the scattered field from the same-size rectangular plate but with the surface impedance $Z_{s v}$. Figure 2 presents the scattered field in the E-plane and $\mathrm{H}$-plane using the fractional integration (in this figure we used order $v=-0.5$ ) and using PO approximation from the impedance plate. Good agreement in the magnitude and phase, particularly near the central region, can be easily observed from these plots. This example suggests that instead of recalculating the scattered field from the impedance plate, one can use the well-known PO results of the scattering from the PEC plate and just apply fractional differintegration on the scattered fields in the far zone in front of the illuminated region, which would provide us with an approximate answer to the far-zone scattering from the impedance plate. So, when we encounter the problem of wave scattering from a finite-size flat plate with a given impedance boundary condition, instead of solving such a problem directly using the PO approximation, one can use the results of PO scattering from PEC version of the same-size same-geometry flat plate, and then apply the fractional differintegration operator on these results. If the variable with respect to which this fractional differintegration is taken is selected appropriately and if the order of the 
fractional differintegration is chosen properly using Eq. (9), then the results of such operation would be approximately similar to the results of scattering from the finite-size impedance plate. We have extended the above case to the situation where we can have two adjacent flat panels and the results support the above idea for the application of fractional operators.

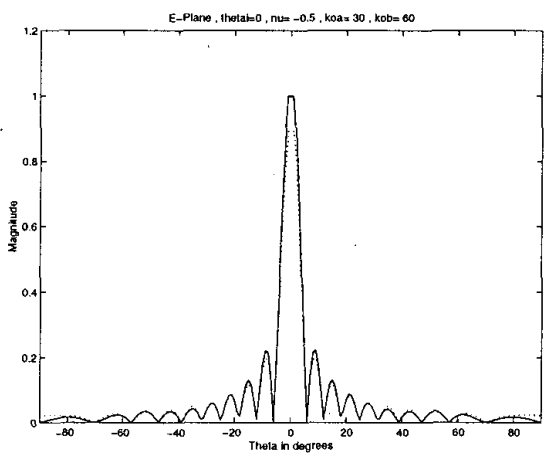

A
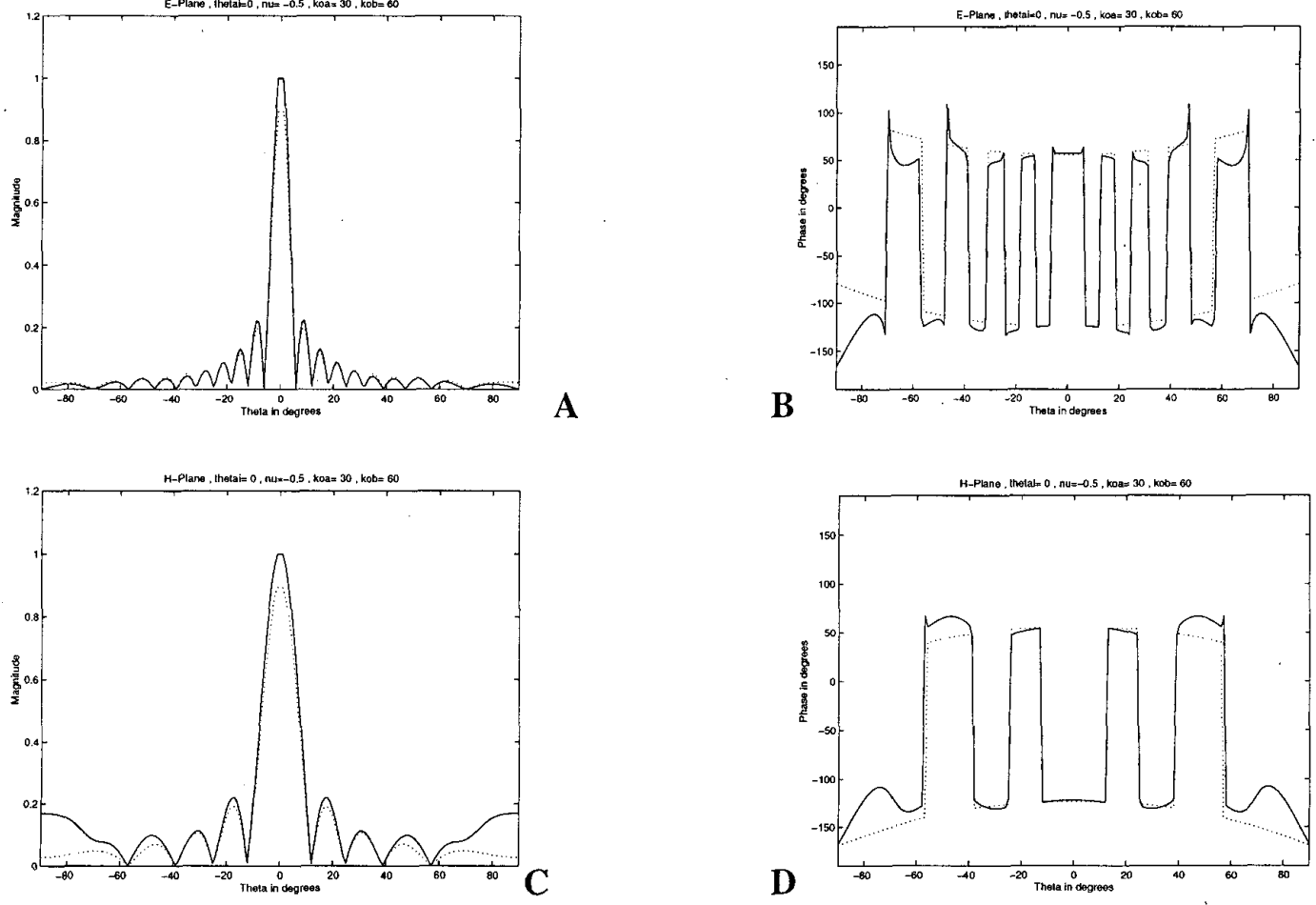

Fig. 2. Scattering of a normally incident EM wave from a rectangular thin plate with dimensions $k_{o} a=30$ and $k_{o} b=60$. First, using the physical optics (PO) approximation, the far-zone scattered field from such a plate with perfectly electric conductor (PEC) surface impedance was obtained. Then we applied the fractional integration operator (with order $v=-0.5$ ) on such far-zone fields in the region in front of the illuminated side of the rectangular plate. The solid lines show the resulting fields from such fractional integration. We then used the impedance transformation given in Eq. (9) to find $Z_{s v}$ for $v=-0.5, \theta_{i}=\theta_{o}=0^{\circ}$ and $Z_{s o}=0$; and afterwards using the PO approximation we directly evaluated the scattered fields from the same-size rectangular plate with this new surface impedance $Z_{s v}$. The dotted lines depict such fields. Panels A and B show the amplitude and phase for these two calculated fields in the E-plane, whereas Panels $C$ and D show the corresponding quantities in the H-plane. Our preliminary analysis shows that the result of fractional differintegration of the scattered fields using PO approximation from the PEC rectangular plate, (solid line), is approximately similar to the scattered field from the same-size rectangular plate but with the surface impedance $Z_{s v}$, (dotted line). Good agreement between the solid line and the dotted line, particularly near the central region, is noticeable.

Kharkov, Ukraine, VIII-th International Conference on Mathematical Methods in Electromagnetic Theory 
In this talk, we will present some of the recent results of our research on applications of fractional operators in radiation and scattering problems and discuss their salient features and physical justifications.

\section{REFERENCES:}

[1] N. Engheta, "Fractional Paradigm in Electromagnetic Theory" a chapter in Frontiers in Electromagnetics, D. H. Werner and R. Mittra (eds.), IEEE Press, New York, chapter 12, pp. 523-552, (2000).

[2] N. Engheta, "On Fractional Paradigm and Intermediate Zones in Electromagnetism: I. Planar Observation," in Microwave and Optical Technology Letters, Vol. 22, No. 4, pp. 236-241 (August 20, 1999).

[3] N. Engheta, "On Fractional Paradigm and Intermediate Zones in Electromagnetism: II. Cylindrical and Spherical Observations," in Microwave and Optical Technology Letters, Vol. 23, No. 2, pp. 100-103 (October 20, 1999).

[4] N. Engheta, "Phase and Amplitude of the Fractional-Order Intermediate Wave" in Microwave and Optical Technology Letters, Vol. 21, No. 5, pp. 338-343 (June 5, 1999).

[5] N. Engheta, "Fractional Curl Operator in Electromagnetics," in Microwave and Optical Technology Letters, Vol. 17, No. 2, pp. 86-91 (February 5, 1998).

[6] N. Engheta, "On Fractional Calculus and Fractional Multipoles in Electromagnetism," IEEE Transactions on Antennas and Propagation, Vol. 44, No. 4, pp. 554-566 (April 1996).

[7] N. Engheta, "Electrostatic "Fractional" Image Methods for Perfectly Conducting Wedges and Cones," in IEEE Transactions on Antennas and Propagation., Vol. 44, No. 12, pp. 1565-1574 (December 1996).

[8] N. Engheta, "On the Role of Fractional Calculus in Electromagnetic Theory" a feature article in IEEE Antennas and Propagation Magazine, Vol. 39, No. 4, pp. 35-46 (August 1997).

[9] N. Engheta, "Use of Fractional Integration to Propose Some "Fractional" Solutions for the Scalar Helmholtz Equation," a chapter in Progress in Electromagnetics Research (PIER) Monograph Series Vol. 12 , Jin A. Kong (ed.), pp. 107-132 (1996).

[10] N. Engheta, "A Note on Fractional Calculus and the Image Method for Dielectric Spheres," Journal of Electromagnetic Waves and Applications, Vol. 9, No. 9, pp. 11791188 (September 1995).

[11] K. B. Oldham and J. Spanier, The Fractional Calculus, Academic Press, New York, (1974).

[12] S. G. Samko, A. A. Kilbas, and O. I. Marichev, Fractional Integrals and Derivatives, Theory and Applications, Gordon and Breach Science Publishers, Langhorne, Pennsylvania, 1993. (Originally published in Russian by Nauka i Tekhnika, Minsk, 1987).

[13] K. S. Miller and B. Ross, An Introduction to the Fractional Calculus and Fractional Differential Equations, John Wiley \& Sons, New York, 1993. 